\title{
Improved human hematopoietic reconstitution in HepaRG co-transplanted humanized NSG mice
}

\author{
Jin Kim ${ }^{1, \#}$, Bokyeong Ryu ${ }^{1, \#}$, Ukjin Kim ${ }^{1}$, Chang-Hwan Kim² ${ }^{2}$ Gyeung-Haeng Hur ${ }^{2}$, C-Yoon Kim ${ }^{3, *} \mathcal{E}_{\text {Jae-Hak Park }}^{1, *}$ \\ ${ }^{1}$ Department of Laboratory Animal Medicine, College of Veterinary Medicine, Seoul National University, Seoul 08826, ${ }^{2} \mathrm{The} 4 \mathrm{th}$ R\&D \\ Institute-6, Agency for Defense Development, Daejeon 34186, ${ }^{3}$ Stem Cell Biology, School of Medicine, Konkuk University, Seoul 05030, \\ Korea
}

\begin{abstract}
Several humanized mouse models are being used to study humanspecific immune responses and diseases. However, the pivotal needs of fetal tissues for the humanized mice model have been huddled because of the demand for ethical and medical approval. Thus, we have verified the hematopoietic and immunomodulatory function of HepaRG and developed a new and easy humanized mouse model to replace the use of fetal liver tissue. HepaRG co-transplanted Hu-NSG mice significantly increased CD45 + lymphocytes and CD19 + B cells and CD3 + T cells than normal Hu-NSG, suggesting enhanced reconstitution of the human immune system. These results have improved the applicability of humanized mice by developing new models easily accessible. [BMB Reports 2020; 53(9): 466-471]
\end{abstract}

\section{INTRODUCTION}

Over the past decades, humanized mouse models with transplanted human cells and tissues have become integral tools in human specific immune research $(1,2)$. Especially, humanized mice produce human cytokine $(3,4)$ and express human major histocompatibility complex types (5). For these reasons, humanized mice have recently been used to study certain human immune diseases including human immunodeficiency virus (HIV) and Epstein-Barr virus $(6,7)$. Recently, a BLT humanized mouse model combining human fetal liver with bone marrow and thymic tissue transplantation has been developed for improved $\mathrm{T}$ cell reconstitution, maturation, and selection (8). Also, it has been reported that the fetal liver is rich in hepatoblasts, hepatocytic precursors and liver sinusoidal endothelial cells thus

${ }^{*}$ Corresponding authors. C-Yoon Kim, Tel: +82-2-3425-0131; Fax: +82-2-455-9012; E-mail: vivavets@gmail.com; Jae-Hak Park, Tel: +822-880-1256; Fax: +82-2-880-1182; E-mail:pjhak@snu.ac.kr

${ }^{\#}$ These authors contributed equally to this work.

https://doi.org/10.5483/BMBRep.2020.53.9.304

Received 14 December 2019, Revised 12 January 2020, Accepted 17 March 2020

Keywords: HepaRG cells, HSC, Humanized mouse, NSG could lead to hematopoietic stem cell engraftment (9).

Liver is an integral organ with a large number and unique distribution of immune cells (10). The liver is also the main hematopoietic space between eight and 24 weeks of gestation, and has all hematolymphoid progenitors, including the erythroid, the myelomonocytic, and the lymphoid lineages (11). Guo et al. transplanted human fetal hepatocytes into humanized mice with transplantation of human fetal thymus and CD34+ hematopoietic stem cell (HSCs). The engrafted human fetal hepatocytes produced IL-3, IL-15, GM-CSF, M-CSF, MCP-1, CXCL-1, and CXCL-10, crucial for immune cell development, differentiation, tissue migration, and retention (10). As such, although fetal livers are an attractive combination for humanized mice, the use of fetal liver tissues have limitations requiring ethical and medical approvals $(12,13)$.

HepaRG cells were isolated from an Edmonson grade I differentiated liver tumor after chronic hepatitis C virus infection, and represent human oval ductular bipotent hepatic cell line. HepaRG cells express OCT3/4, a marker of embryonic stem cells (14), and express oval cell-specific markers M2-PK, OV-1, OV-6, CD34, NCAM, ABCG2, CK18, and CK19 $(15,16)$. In particular, they also express HSC markers such as CD34, Thy1, Flt-3, c-Kit, IL-3R $\alpha$, and LIF-R, and lymphoid and myeloid markers such as CD13, CD33, and CD10 (16). These characters suggest that HepaRG could play an alternative role for the fetal liver to reconstitute the human immune system in humanized mice.

In this study, we have developed an advanced humanized mouse model without human fetal tissue. We hypothesized that engrafted CD34+ HSC with HepaRG cells could reconstitute an improved human immune system in NOD.Cg-Prkdc $c^{\text {cid }} / / 2 r^{\text {tm }}{ }^{\text {Wil }} /$ SzJ (NOD-scid IL2rg ${ }^{\text {null }}$, NSG) mice. Based on the next generation sequencing (NGS) analysis of HepaRG, we confirmed the human $\mathrm{B}, \mathrm{T}$ cell population resulted from HSC differentiation by HepaRG coculture and confirmed the reconstitution of the improved human immune system in NSG mice. In this paper, we demonstrate the feasibility of a new model of humanized mice via HepaRG transplantation.

ISSN: 1976-670X (electronic edition)

Copyright (C) 2020 by the The Korean Society for Biochemistry and Molecular Biology

(c) This is an open-access article distributed under the terms of the Creative Commons Attribution Non-Commercial License (http://creativecommons.org/licenses/by-nc/4.0) which permits unrestricted non-commercial use, distribution, and reproduction in any medium, provided the original work is properly cited. 


\section{RESULTS}

\section{Analysis of HepaRG cells}

HepaRG cells were analyzed for the detection of CD34 + population and were shown to have $\sim 5 \%$ population (Fig. 1A). Additionally, gene expression of HepaRG cells were analyzed compared with human dermal fibroblasts and the enrichment and crosslink figures of gene ontologies were drawn (Fig. 1B, C). The HepaRG cells showed significantly higher levels of gene expressions than the human dermal fibroblasts within the categories of T cell mediated immunity, antimicrobial humoral response, antibacterial humoral response, positive regulation by host of viral process, ribosome biogenesis, ribosomal large subunit biogenesis, translational elongation, and detoxification.

\section{Differentiation of HSCs into lymphoid lineages}

The HSCs were co-cultured with human mesenchymal stem

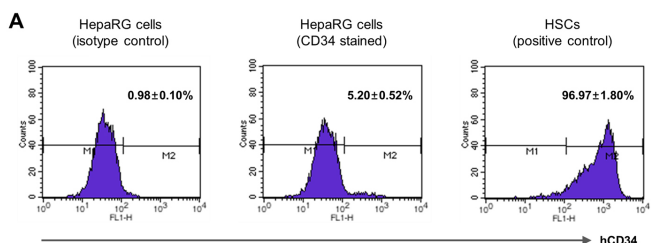

B
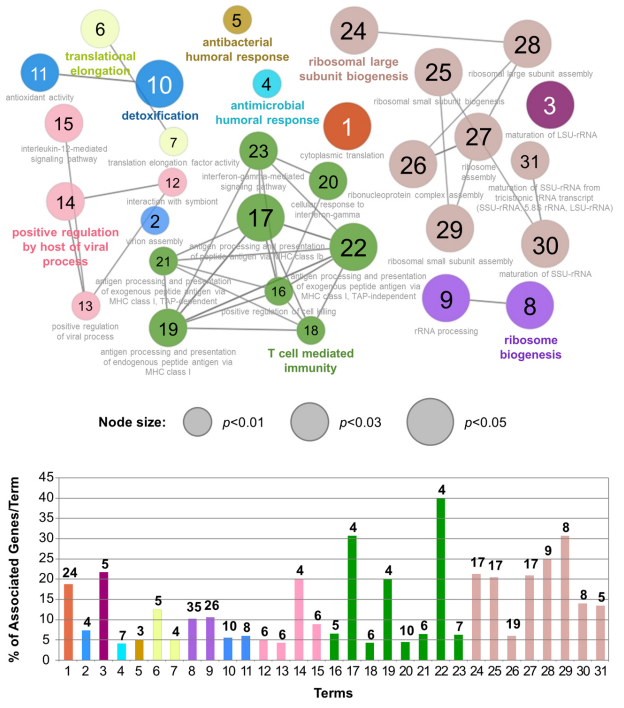

Fig. 1. Characterization of the CD34+ HepaRG cells and the biological processes of the enriched genes. (A) Flow cytometry analysis of the HepaRG cells for the detection of hCD34+ population using the isotype control and anti-hCD34 antibodies. The HSCs were used as a positive control. (B) The biological processes enriched amongst genes in the HepaRG cells than human dermal fibroblasts are shown in an ClueGO enrichment map and are numbered 1-31. (C) The percentage of associated genes per term for each node in (B) are shown. Genes included in each node are listed in the supplementary material and the number of genes is represented on top of each bar. cells (hMSCs) under treatment of HepaRG supernatant concentrates for two weeks (Fig. 2A). Immunostaining for HSCs was performed two weeks after co-cultures by staining for the human CD45 (lymphoid-lineage marker), the human CD3 (early $\mathrm{T}$ cell marker), and the human CD19 (early B cell marker) (Fig. 2B, C). After two-week co-culture, the hCD $45^{\text {low }}$ population increased especially in the HepaRG-concentrate-treated group (50.06 $\pm 4.33 \%)$. Additionally, the naïve growth media-treated and the HepaRGconcentrate-treated groups showed dramatically increased hCD3+ population $(72.00 \pm 7.02 \%$ and $77.90 \pm 5.38 \%$, respectively) than the control group $(19.28 \pm 1.13 \%)$ in the $\mathrm{hCD} 45^{\text {low }}$ population, and increased hCD19+ population $(46.47 \pm 1.97 \%$ and $49.57 \pm 8.38 \%$, respectively) than the control group $(25.90 \pm$ $1.74 \%$ ) in the hCD $45^{\text {high }}$ population.

\section{Transplantation analysis of HepaRG cells}

To confirm the transplantation, the Dil-labeled HepaRG cells were transplanted into humanized NSG (Hu-NSG) mice and analyzed on day three, seven, and 14 (Fig. 3A). The HepaRG labeled with Dil showed red fluorescence, and nuclei were counterstained with dapi (blue) (Fig. 3B). The cells were detected around the liver portal vein at the day three. On the day seven, the cells were well aggregated and maintained and slightly

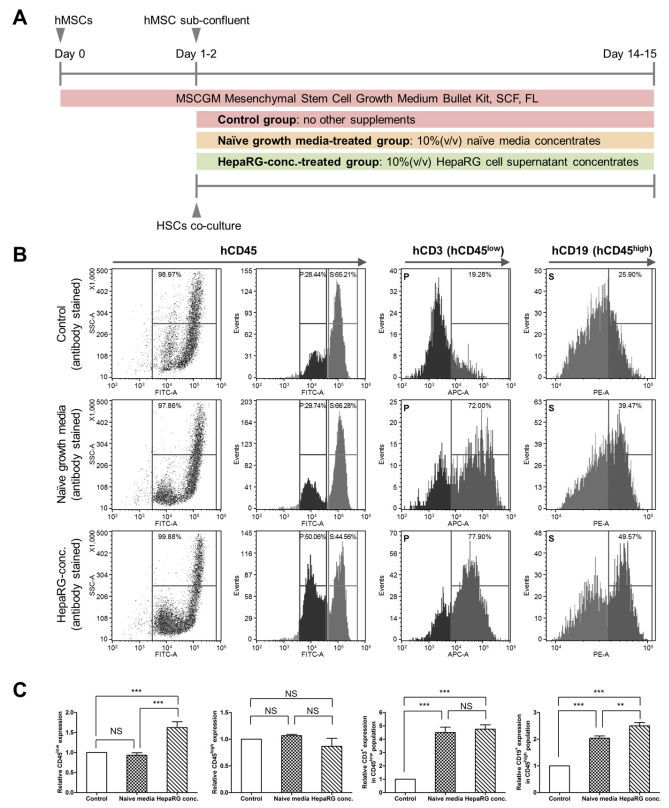

Fig. 2. Co-culture and characterization of HSCs. The HSCs were cocultured with hMSCs and analyzed with flow cytometry. (A) The description of the co-culture schemes. (B) The co-cultured HSCs were analyzed using the anti-hCD45, anti-hCD3, anti-hCD19 antibodies. (C) The relative expressions of $\mathrm{hCD} 45^{\text {low }}, \mathrm{hCD} 45^{\text {high }}, \mathrm{hCD} 3+$ in $\mathrm{CD} 45^{\text {low }}$ population, and $\mathrm{hCD} 19+$ in $\mathrm{CD} 45^{\text {high }}$ population were calculated. Data shown are mean \pm SD (NS, not significant; $* * P<0.01$; $* * * P<0.001)$ 
A

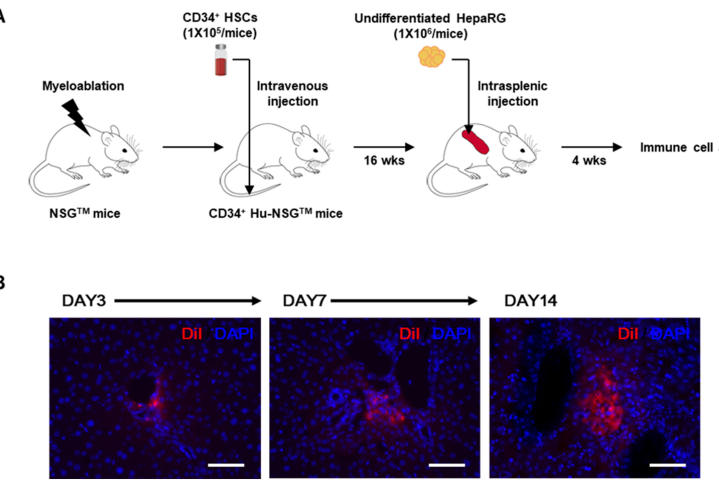

Fig. 3. Generation of humanized mice with HepaRG transplantation. The HepaRG cells were transplanted into humanized mice and confirmed by Dil staining at each time point. (A) The description of the experimental schemes. (B) The transplanted Dil-labeled HepaRG cells (red fluorescence) counterstained with dapi nuclei staining (blue) were detected at day three, seven, and 14. Scale bar $=50 \mu \mathrm{m}$

scattered compared with the day three. After day 14, the cells around the portal veins exhibited a red fluorescent signal without dispersion and successfully engrafted.

\section{HepaRG co-transplanted Hu-NSG mice show promoted} human CD45 + CD19 + and CD45 + CD3 + cell formation

HepaRG cells were transplanted into Hu-NSG mice, and CD45 + hematopoietic cells were examined in the blood, spleen, and bone marrow. The level of human CD45+ hematopoietic cell significantly increased in the blood of HepaRG co-transplanted Hu-NSG mice $(88.1 \pm 3.4 \%)$ compared with Hu-NSG without HepaRG $(76.0 \pm 5.0 \%$ ) (Fig. 4A). In the spleen and bone marrow, the percentages of human CD45+ cells were $68.2 \pm 8.0 \%$ and $65.9 \pm 3.6 \%$ when administered CD34 + cells only, while 1.3 and 1.2 folds increased in HepaRG co-transplanted Hu-NSG mice $(88.3 \pm 3.6 \%$ and $81.2 \pm$ $5.1 \%$, respectively) (Fig. 4A).

Additionally, the development of human CD19+ B cells and CD3 + T cells was monitored in the blood, spleen and bone marrow (Fig. 4B). Similar as the increase in CD45+ hematopoietic cells, the percentages of human CD45+CD19+ population were higher in the HepaRG co-transplanted HuNSG mice. The levels increased 1.9-fold $(27.5 \pm 4.3 \%)$ in blood, 1.5 -fold $(73.9 \pm 5.4 \%)$ in spleen, and 1.2-fold $(68.6 \pm 7.51 \%)$ in bone marrow (Fig. 4C). Likewise, the percentages of human CD45+CD3 + population were increased in all samples. Although the levels only showed tendency to increase in the blood $(P=0.05)$, they were statistically significant in the spleen and bone marrow. The levels were 1.5-fold (14.6 $\pm 2.0 \%)$ and 3.1 -fold $(3.0 \pm 2.2 \%)$ higher, respectively. These results indicate that the HepaRG cells transplanted into the Hu-NSG mice support rapid engraftment and differentiation of human lymphohematopoietic cells.
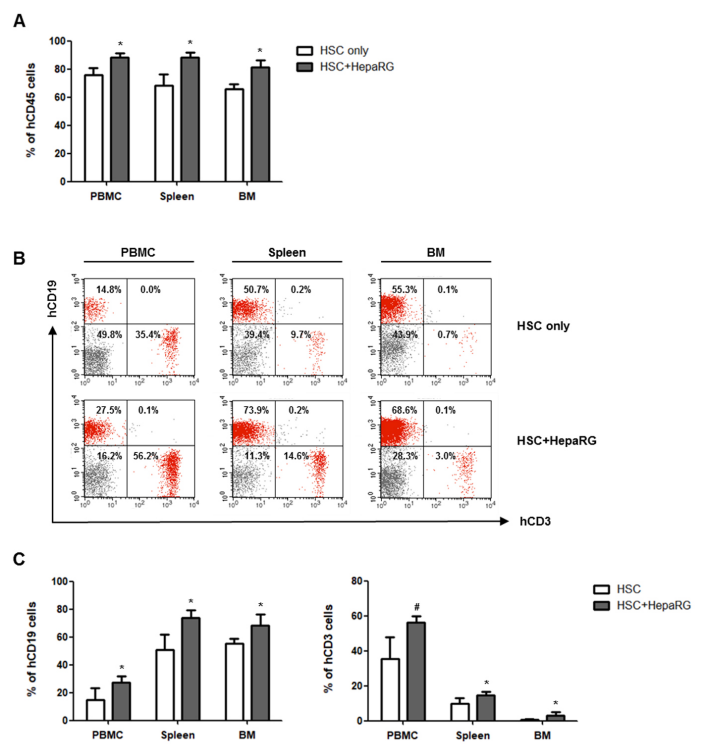

Fig. 4. Reconstitution of human lymphocytes in the blood, spleen, and bone marrow. The peripheral blood mononuclear cell (PBMC), spleen, and bone marrow (BM) were analyzed using animals 20 weeks after the HSC transplantation. The percentages of human CD45 + lymphocytes, CD19+ B and CD3 + T cells were assessed according to if the HepaRG cells were transplanted using the flow cytometry analysis. (A) The percentage and absolute count of the human lymphocytes $(\mathrm{CD} 45+)$. (B) The distribution (Gated on $\mathrm{CD} 45+)$ and $(\mathrm{C})$ The percentage of $\mathrm{B}$ cells $(\mathrm{CD} 19+)$ and $\mathrm{T}$ cells $(\mathrm{CD} 3+)$. Data shown are mean $\pm \mathrm{SD}\left({ }^{*} \mathrm{P}<0.05\right.$; $\left.{ }^{*} \mathrm{P}=0.05\right)$.

\section{DISCUSSION}

In this study, we have developed the HepaRG co-transplanted $\mathrm{Hu}$-NSG mice that can replace the use of human fetal liver tissue with the HepaRG cells.

NGS analysis was performed to confirm the hematopoietic and immunomodulatory properties of HepaRG, and it was confirmed that the characteristics of T cell and humoral immunity were highly expressed. The genes involve in Rap1 signaling pathway (KEGG hsa04015) (17), HIF-1 signaling pathway (hsa 04066) (18), endocytosis (hsa04144), Lysosome (hsa04142), antigen processing and presentation (hsa04612), and IL-17 signaling pathway (hsa04657) (19), which regulate lymphocytic differentiation and function. The HepaRG was also previously reported to secrete aplenty bioactive molecules related to regulation of humoral and cytotoxic immune responses (20). These data imply that the HepaRG has immune differentiation regulating function similar to human fetal liver function. Also, the hepatocarcinoma derived cells, such as HepaRG and HepG2, have been reported to have CD34 positive population (Fig. 1A) (21) and to support human embryonic and induced pluripotent stem cells differentiate into the hematopoietic lineage (22). Although the hematopoietic activity was not directly 
classified in our NGS analysis, these results suggest that the HepaRG may contribute to the establishment of hematopoietic and immune functions.

Based on the results of the NGS and secretome, to confirm that HepaRG affects immune cell differentiation of HSC, the concentrated culture medium of HepaRG was added to the conventional MSC co-culture differentiation method to induce the immune cell differentiation of the HSC. The concentrated culture medium of the HepaRG significantly affected the population of the $C D 45^{\text {low }}$ cells which are innate phagocytic cells (23), and increased population of the $\mathrm{CD} 45^{\text {low }} \mathrm{CD} 3+$ cells and the $\mathrm{CD} 45^{\text {high }} \mathrm{CD} 19+$ cells demonstrated that the HSCs have been promoted differentiation into T-lymphocytes and B-lymphocytes, respectively. These results can be interpreted in that the immune regulating properties of the HepaRG shown in the $\mathrm{GO}$ analysis influenced HSC differentiation through secretome in the concentrated culture medium.

However, in vitro experiments were difficult to control for the long-term culture and further differentiation condition. To confirm this, we transplanted the HepaRG cells into humanized mice by intrasplenic injection and investigated the differentiation of transplanted HSCs in in vivo level. According to previous studies (24), the undifferentiated HepaRG are transplanted into immunodeficient mice and then differentiated into albuminproducing hepatocytes. We administered the HepaRG 16 weeks after HSC transplantation, considering the in vivo maturation period of $B$ and $T$ cells in the humanized mice (25), and confirmed the population of immune cells in blood, bone marrow, and spleen after approximately four weeks. The injected HepaRG cells via the spleen migrated and engrafted into the liver. This means that the differentiation of the HSCs administered through the vein is not a direct contact effect of the HepaRG, but the paracrine effect; this is consistent with the mechanism of the differentiation effect of HSCs by HepaRG secretion in vitro.

Interestingly, significant increase in CD45 + cells was observed in the group co-transplanted with the HepaRG compared to the Hu-NSG group. This is presumed to be because of the absence of an induction environment in vivo, unlike in vitro experiments in which hMSCs induce differentiation of HSCs, meaning that the transplanted HepaRG cells were effective for differentiating CD34 + HSCs into CD45 + cells. Also, the CD19+ and $\mathrm{CD} 3+$ cells also increased significantly, indicating that the transplanted HepaRG promoted HSC differentiation into B and $T$ cells in vivo. Although inconsistent with the results of Guo et al. in which the reconstitution of immune cells in the liver significantly improved depending on the human hepatocyte implantation (10), our results show significant lymphohematopoietic changes in blood, spleen, and bone marrow. Based on our in vitro RNA-sequencing and secretome analysis results, these can be interpreted that the place wherein hematopoiesis occurs was changed because of the types of cell used or the paracrine effects of the cell were different. Our results show that the transplantation of the HepaRG is effective for reconstructing the human immune system of humanized mice.
In conclusion, we have verified the hematopoietic and immunomodulatory function of the HepaRG and developed a new and easy humanized mouse model to replace the use of fetal liver tissue. The HepaRG co-transplanted Hu-NSG mice had significantly increased CD45 lymphocytes, CD19 B cells, and CD3 T cells, than the normal Hu-NSG, suggesting enhanced reconstitution of the human immune system. These results have improved the applicability of humanized mice by developing new models easily accessible to researchers in the field of human immune-specific disease research.

\section{MATERIALS AND METHODS}

\section{Cell culture}

The HepaRG cells (Biopredic International, Saint Grégoire, France), derived from a human hepatocellular carcinoma, were maintained in William's Medium E (Thermo Fisher Scientific, MA, USA) supplemented with $10 \%$ FBS, 100 units $/ \mathrm{mL}$ penicillin, $100 \mu \mathrm{g} / \mathrm{mL}$ streptomycin, ITS $(2 \mu \mathrm{g} / \mathrm{mL}$ insulin, $1.1 \mu \mathrm{g} / \mathrm{mL}$ transferrin, $1.34 \mathrm{ng} / \mathrm{mL}$ sodium selenite; Thermo Fisher Scientific), $1 \%$ GlutaMAX ${ }^{\mathrm{TM}}-\mathrm{I}$ (Thermo Fisher Scientific), and $8.65 \mu \mathrm{M}$ hydrocortisone hemisuccinate (growth medium). The undifferentiated cells were seeded at a density of $3.2 \times 10^{5} \mathrm{cells} / \mathrm{cm}^{2}$ into flat-bottom multi-well plates. Cells were maintained for two weeks and the medium was replaced every other day. For differentiation, when the cells were highly confluent, they were incubated in the growth medium added with $1.7 \%$ DMSO (differentiation medium) for two more weeks. In the differentiation process, the medium was replaced every two or three days. All cultures were performed at $37^{\circ} \mathrm{C}$ in $95 \%$ humidity with $5 \% \mathrm{CO}_{2}$ condition. Cultured cells were analyzed with flow cytometry for the detection of hematopoietic potentials. The analyses were conducted in triplicate.

\section{RNA extraction and sequencing}

The NGS was conducted to compare gene expression levels in the HepaRG cells to human dermal fibroblasts (hDFs). The hDFs were chosen as normal control human cells. For this purpose, the total RNA was extracted from the cells using GeneAII ${ }^{\mathbb{R}}$ Hybrid- $R^{\mathrm{TM}}$ (Seoul, Korea) according to the manufacturer's protocol. All RNA samples were of high and comparable quality as determined by the Agilent 2100 Bioanalyzer System (Agilent, Böblingen, Germany). cDNA libraries were generated according to standard procedures using a Lexogen QuantSeq 3' mRNA-Seq Library Prep Kit (Lexogen, Vienna, Austria). Libraries were sequenced on an Illumina NextSeq500 (Illumina, CA, USA) in single-end (SE) 75-base mode. Each set of RNA-seq data was derived from the triple biological samples.

The illumina reads were trimmed to only retain reads with values higher than the average Q20 in Phred quality score using BBDuk (part of BBtools). The remaining reads were mapped to the respective reference genome sequences (hg19, genome database: USCS) using Bowtie2. The calculation reads were counted using Bedtools (https://bedtools.readthedocs.io/en/latest). 
We then quantified and quantile normalized gene expression levels using the edgeR. The read mapping and expression quantification were performed separately for each sample. The significant genes were selected (normalized data $>4$ and fold change $>2$ ) and analyzed using ClueGO v2.5.5, and Clue Pedia v1.5.5 on Cytoscape v3.7.2 on Java 1.8.0 162 (P value $<$ 0.05, GO tree interval level 4-6, Kappa score 0.4).

In the ClueGO results, only significantly enriched gene ontologies $(\mathrm{GOs})$ were present $(\mathrm{P}<0.05)$ as nodes (circles with colors). Each node represents a significantly enriched GO of the biological processes and the node size reflects the enrichment significance of the terms. The nodes with the same color indicate biological processes that share genes. The percentages of the genes from the list that were associated with the term, compared with all the genes associated with the term, were also calculated.

\section{Concentration of HepaRG supernatants}

The supernatants collected from the HepaRG cell culture were pooled and concentrated with the Amicon Ultra-15 centrifugal filter units (MWCO $10 \mathrm{kDa}$; Merck Millipore, MA, USA). Briefly, the HepaRG supernatants were loaded into the filter devices and centrifuged at 4500 RPM for 20 minutes to be concentrated at one-tenth in volume. The concentrates of the supernatants were used $10 \%(\mathrm{v} / \mathrm{v})$ in the culture media for co-cultures of the HSCs and the hMSCs. The naïve growth media without the HepaRG cell culture were used as a control for the HepaRG cell supernatant and were concentrated with the same procedures.

\section{Co-cultures for the HSCs on the hMSCs with treatment of HepaRG supernatant concentrate}

Co-cultures of the HSCs on hMSCs were performed as previously described (26). Briefly, the hMSC were seeded in 12-well plates one or two days before setting up the co-cultures. The HSCs $\left(2 \times 10^{3}\right.$ cells/well) were plated on sub-confluent hMSC layers using MSCGM Mesenchymal Stem Cell Growth Medium Bullet Kit (Lonza, Basel, Switzerland) in the presence of $10 \mathrm{ng} / \mathrm{mL}$ SCF (Peprotech, NJ, USA) and $5 \mathrm{ng} / \mathrm{mL}$ FL (Peprotech). The cells of some wells were treated with $10 \%(\mathrm{v} / \mathrm{v})$ HepaRG supernatant concentrates or $10 \%(\mathrm{v} / \mathrm{v})$ naïve growth medium concentrates while cells of the control group were cultured in naïve MSCGM. Half of the culture medium was replaced with fresh medium containing the same cytokines twice a week for two weeks. Cultured cells were analyzed with flow cytometry for the detection of lymphoid differentiation. The analyses were conducted in triplicate.

\section{Mice}

Mouse husbandry and experiments were conducted with the approval of the Seoul National University Institutional Animal Care and Use Committee (SNU-171024-8-5). The Hu-NSG mice injected with CD34+ HSCs after myeloablation were purchased from The Jackson Laboratory (Bar Harbor, ME, USA). All animals were kept in specific pathogen free facilities in individually ventilated cages, with free access to autoclaved water and food, and 12:12 h dark/light cycles.

\section{Generation of humanized mice with/without HepaRG transplantation}

The HepaRG cells were transplanted to 19-week old female Hu-NSG mice (16 weeks after CD34+ HSCs implantation, Donor ID 5777; 5847; 6170) through intrasplenic injection. The HepaRG cells were washed with culture medium, centrifuged, and resuspended. For anesthesia, mixture of alfaxan (Careside, Gyeonggi-do, Korea) and xylazine (Bayer Korea, Seoul, Korea) was administered intraperitoneally. After the skin and peritoneum incision, the spleen was exposed and $1 \times 10^{6}$ HepaRG cells were transplanted. Silk sutures and Surgifit sutures (Ailee, Busan, Korea) were used to close the peritoneum and skin, respectively.

\section{Transplantation analysis}

The HepaRG cells were incubated with CellTracker ${ }^{\mathrm{TM}} \mathrm{CM}$-Dil (Thermo Fisher Scientific) diluted in culture media for 24 hours and then used for transplantation. Animals were euthanized three, seven, and 14 days after the HepaRG transplantation. The spleen was subsequently removed and fixed in 4\% PFA solution (Biosesang, Gyeonggi-do, Korea) for 4-24 hours at room temperature. After the incubation with $30 \%$ sucrose solution until sink to the bottom, the tissue was transferred to the mold filled with OCT compound (Sakura Finetek, CA, USA). The OCT-embedded tissue was frozen, sectioned in 7 $\mu \mathrm{m}$ thickness using cryostat (Leica Microsystems, Germany), and mounted on slide glass (O. Kindler, $\mathrm{GmbH}$, Germany). The tissue was fixed in cold acetone for 20 minutes, washed with PBS, and stained with DAPI solution (Sigma-Aldrich, MO, USA) to confirm the transplantation.

\section{Antibodies and flow cytometry analysis (FACS)}

The HepaRG cells, HSCs from co-cultures, and engrafted hematopoietic cells were analyzed with fluorophore-linked primary antibodies purchased from eBiosciences (San Diego, CA, USA), or Biolegend (San Diego, CA, USA). The following antibodies (clones) were used: FITC anti-human CD34 antibody (561), FITC anti-human CD45 antibody (HI30), APC CD3 monoclonal antibody (SK7), PE CD19 monoclonal antibody (HIB19), FITC CD4 monoclonal antibody (SK3), and PE CD8a monoclonal antibody (SK1). Single cell suspensions were prepared from the spleen and the bone marrow (obtained from the femur) of mice and whole blood was collected in heparin. Single cell suspensions and whole blood were washed with PBS and FACS buffer (PBS with 3\% bovine serum albumin). Cells were then incubated with antibodies for 45 minutes at $4^{\circ} \mathrm{C}$ in the dark. Samples were washed with FACS buffer and fixed with paraformaldehyde. Events $\left(10^{4}\right.$ to $\left.10^{6}\right)$ were collected per sample and analyzed using BD FACSCalibur (BD Biosciences, San Jose, CA, USA). The proportion of cells was calculated by flow cytometry using BD CellQuest Pro software (BD Biosciences). The FACS analyses were conducted in triplicate in vitro and $\mathrm{n}=5$ and 4 animals were analyzed for HSC only and the HepaRG co-transplanted group, respectively. 


\section{Statistical analyses}

Statistical analyses were performed using the GraphPad PRISM software version 5 (GraphPad, San Diego, CA). An unpaired t-test was performed to determine statistically significant differences between the mean values of each data.

\section{ACKNOWLEDGEMENTS}

This study was supported by the Korea Agency for Defense Development under contact No. UD170032ID and the National Research Foundation of Korea [Grant/Award Number: NRF-2018R1C1B6007 354]. Further support was also provided by the Research Institute of Veterinary Science, and the BK21 PLUS Program for Creative Veterinary Science Research, College of Veterinary Medicine, Seoul National University.

\section{CONFLICTS OF INTEREST}

The authors have no conflicting interests.

\section{REFERENCES}

1. Lan $\mathrm{P}$, Tonomura $\mathrm{N}$, Shimizu A, Wang $\mathrm{S}$ and Yang YG (2006) Reconstitution of a functional human immune system in immunodeficient mice through combined human fetal thymus/liver and CD34 + cell transplantation. Blood 108, 487-492

2. Lavender KJ, Pang WW, Messer RJ et al (2013) BLThumanized C57BL/6 Rag2 - I- $\gamma \mathrm{C}-/-\mathrm{CD} 47-/-$ mice are resistant to GVHD and develop B-and T-cell immunity to HIV infection. Blood 122, 4013-4020

3. Rongvaux A, Willinger T, Martinek J et al (2014) Development and function of human innate immune cells in a humanized mouse model. Nat Biotechnol 32, 364

4. Saito Y, Ellegast JM, Rafiei A et al (2016) Peripheral blood CD34 + cells efficiently engraft human cytokine knock-in mice. Blood 128, 1829-1833

5. Shultz LD, Saito Y, Najima Y et al (2010) Generation of functional human T-cell subsets with HLA-restricted immune responses in HLA class I expressing NOD/SCID/IL2rynull humanized mice. Proc Natl Acad Sci U S A 107, 1302213027

6. Klein F, Halper-Stromberg A, Horwitz JA et al (2012) HIV therapy by a combination of broadly neutralizing antibodies in humanized mice. Nature 492, 118

7. Yajima M, Imadome KI, Nakagawa A et al (2008) A new humanized mouse model of Epstein-Barr virus infection that reproduces persistent infection, lymphoproliferative disorder, and cell-mediated and humoral immune responses. J Infect Dis 198, 673-682

8. Lan P, Tonomura N, Shimizu A, Wang S and Yang YG (2006) Reconstitution of a functional human immune system in immunodeficient mice through combined human fetal thymus/liver and CD34+ cell transplantation. Blood $108,487-492$

9. Fomin ME, Beyer Al and Muench MO (2017) Human fetal liver cultures support multiple cell lineages that can engraft immunodeficient mice. Open Biol 7, 170108
10. Guo J, Li Y, Shan Y et al (2018) Humanized mice reveal an essential role for human hepatocytes in the development of the liver immune system. Cell Death Dis 9, 667

11. Touraine JL (1983) Bone-Marrow and Fetal-Liver Transplantation in Immunodeficiencies and Inborn Errors of Metabolism: Lack of Significant Restriction of T-Cell Function in Long-Term Chimeras Despite HLA-Mismatch. Immunol Rev 71, 103-121

12. Kapp MB (2006) Ethical and legal issues in research involving human subjects: do you want a piece of me? J Clin Pathol 59, 335-339

13. Geraghty R, Capes-Davis A, Davis J et al (2014) Guidelines for the use of cell lines in biomedical research. Br J Cancer 111, 1021

14. Kubo T, Kuroda Y, Hojyo M et al (2016) Maintenance of hepatic progenitor-like characteristics of HepaRG cells by cultivation on VECELL Inserts. ALTEX 21, 71-79

15. Parent $R$, Marion MJ, Furio $L$, Trépo $C$ and Petit MA (2004) Origin and characterization of a human bipotent liver progenitor cell line. Gastroenterology 126, 1147-1156

16. Cerec V, Glaise D, Garnier D et al (2007) Transdifferentiation of hepatocyte-like cells from the human hepatoma HepaRG cell line through bipotent progenitor. Hepatology 45, 957-967

17. Mou F, Praskova M, Xia F et al (2012) The Mst1 and Mst2 kinases control activation of rho family GTPases and thymic egress of mature thymocytes. J Exp Med 209, 741-759

18. Clambey ET, McNamee EN, Westrich JA et al (2012) Hypoxia-inducible factor-1 alpha-dependent induction of FoxP3 drives regulatory T-cell abundance and function during inflammatory hypoxia of the mucosa. Proc Natl Acad Sci U S A 109, E2784-2793

19. Qian Y, Kang Z, Liu C and Li X (2010) IL-17 signaling in host defense and inflammatory diseases. Cell Mol Immunol 7, 328-333

20. Tascher G, Burban A, Camus S et al (2019) In-Depth Proteome Analysis Highlights HepaRG Cells as a Versatile Cell System Surrogate for Primary Human Hepatocytes. Cells 8, 192

21. Jabari S, Meissnitzer M, Quint K et al (2009) Cellular plasticity of trans- and dedifferentiation markers in human hepatoma cells in vitro and in vivo. Int J Oncol 35, 69-80

22. Lu M, Kardel MD, O'Connor MD and Eaves CJ (2009) Enhanced generation of hematopoietic cells from human hepatocarcinoma cell-stimulated human embryonic and induced pluripotent stem cells. Exp Hematol 37, 924-936

23. Rangaraju S, Raza SA, Li NX et al (2018) Differential Phagocytic Properties of CD45(low) Microglia and CD45 (high) Brain Mononuclear Phagocytes-Activation and AgeRelated Effects. Front Immunol 9, 405

24. Higuchi Y, Kawai K, Yamazaki H et al (2014) The human hepatic cell line HepaRG as a possible cell source for the generation of humanized liver TK-NOG mice. Xenobiotica 44, 146-153

25. Audige A, Rochat MA, Li D et al (2017) Long-term leukocyte reconstitution in NSG mice transplanted with human cord blood hematopoietic stem and progenitor cells. BMC Immunol 18, 28

26. Ichii M, Oritani K, Yokota T et al (2010) Stromal cell-free conditions favorable for human B lymphopoiesis in culture. J Immunol Methods 359, 47-55 